\title{
Renal Infarction: The Forgotten Cause of Abdominal Pain
}

\author{
Sabah Meryem, Khalfaoui Mohamed Amine, El oury Hiba, Jabrane Fatimzahra, Kassimi Mariam, Habi Jihane \\ Department of Nephrology, International University Hospital Sheikh Khalifa, Mohammed VI University of Health Sciences (UM6SS) \\ Department of Radiology, International University Hospital Sheikh Khalifa, Mohammed VI University of Health Sciences (UM6SS)
}

DOI: 10.29322/IJSRP.11.11.2021.p11955

http://dx.doi.org/10.29322/IJSRP.11.11.2021.p11955

\begin{abstract}
Renal infarction is a rare under-reported condition and is rarely diagnosed at presentation. As it can mimic other conditions, such as nephrolithiasis and pyelonephritis, the diagnosis is often delayed or missed. Unfortunately, this leads to late treatment initiation and therefore may result in irreversible kidney damage. The most common causes are thromboembolism and atheromatous disease.

We present three case reports of renal infarction with similar clinical presentation although distinctive etiologies and evolution. The first one presenting in a context of pulmonary embolism, the second one is reported in a patient with Ehlers-Danlos syndrome, and the third one after surgical valve repair
\end{abstract}

Index terms- Renal infarction, Ehlers-Danlos syndrome, pulmonary embolism, valve repair

\section{INTRODUCTION}

Renal infarction is a pathological condition which is caused by renal artery occlusion that may lead to permanent renal damage. It is a rare under-reported condition and is rarely diagnosed at presentation. The nonspecific clinical presentation, frequently like other renal manifestations such as nephrolithiasis and pyelonephritis, is the main factor responsible for delay in diagnosis. Unfortunately, this leads to late treatment initiation and therefore may result in prolonged ischemia and development of irreversible kidney damage. Thromboembolism and atheromatous diseases are the usual causes of renal infarction (1). Nevertheless, other etiologies are described in the literature such as autoimmune diseases and hypercoagulable states. The authors present three cases of renal infarcts presenting initially with acute abdominal pain but with distinct causes, which underlines the importance of medical thoroughness in both patient history and warning clinical signs.

\section{CASE REPORT}

A. $\quad$ Case 1

A 62-year-old female patient with a known history of myeloproliferative syndrome presenting at the emergency department with severe respiratory distress. The patient had no other comorbidities such as hypertension, diabetes mellitus, This publication is licensed under Creative Commons Attribution CC BY. coagulation disorders, renal or heart disease. On physical examination, the patient was in a poor general state, the temperature was $99.1^{\circ} \mathrm{F}$, blood pressure was $110 / 75 \mathrm{mmHg}$, heart rate was $62 \mathrm{bpm}$ and respiratory rate was $36 / \mathrm{min}$. She presented severe chest pain and dyspnea. CT angiography revealed pulmonary embolism. The patient was immediately admitted for surveillance and received

anticoagulation therapy. Twenty-four hours later, the patient reported acute left flank pain. Blood analysis revealed leukocytosis $(18.41 \times 103 / \mu \mathrm{L}$ with $81.8 \%$ neutrophils $)$, modest elevation of transaminases (aspartate transaminase $134 \mathrm{U} / \mathrm{L}$, alanine transaminase $142 \mathrm{U} / \mathrm{L}$ ) and frank elevation of lactate dehydrogenase (LDH) - $885 \mathrm{U} / \mathrm{L}$ (reference value 135 - $225 \mathrm{U} / \mathrm{L}$ ). Cytobacteriological testing of urine was normal. Abdominal ultrasound revealed liver no renal nor spleen abnormalities. An abdominal CT was performed revealing a hypodense peripheral triangle-shaped area in the left kidney (Fig1). According to Vascular Surgery's opinion, there was no indication for interventional therapy at that time, nor for additional drugs as the patient was already receiving anticoagulation therapy. She was discharged after two weeks with no kidney damage. Presently the patient is on chronic coumarin therapy and under surveillance on Cardiology and Hematology consultation. Biological tests showed renal normal function.

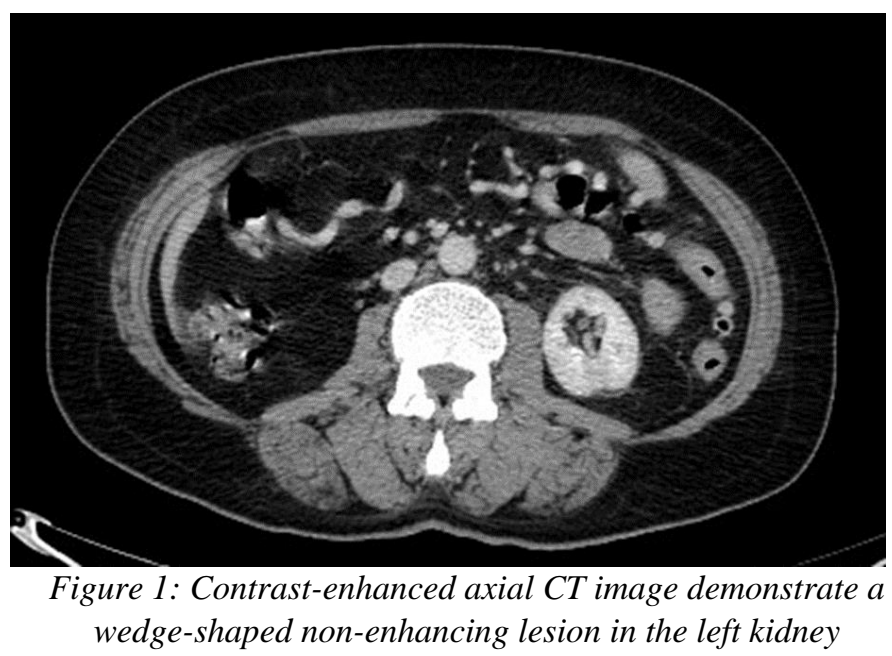


A 40 years old male patient with a known history of a mutation in COL3A1 gene associated with vascular Ehlers-Danlos syndrome, referred to the emergency department for sudden onset of abdominal pain with 16 hours of evolution, located in the upper quadrant and right flank, concomitant with nausea, vomiting and fever. On examination, the patient was agitated, his temperature was $101.3{ }^{\circ} \mathrm{F}$, heart rate was $112 \mathrm{bpm}$, and blood pressure was 98/71 mmHg. Abdominal examination was negative for tenderness or any other abnormality. White blood cell count was elevated at 14600 cells per cubic millimeter. Urinalysis showed 3 white blood cells per field and no hematuria. Non-contrast abdominal computed tomography showed a triangular hypodense area in the right kidney, initially interpreted as "severe pyelonephritis". The patient received antibiotics without clinical improvement. In the absence of an evident sign of a urinary tract infection, a CT angiogram was pursued as a renal infarction was highly suspected. It revealed a dissection of the right renal artery with subsequent infarction in the inferior pole of the right renal parenchyma (Fig 2). Antibiotic therapy was withheld and the patient received betablockers and heparin with symptom resolution after three days of anticoagulation. An etiological study was conducted, including electrocardiogram (ECG) and echocardiography in search for a cardiopathy, as well as a thrombophilia assay. No abnormality was noted. At 3 months follow-up, the patient presented normal renal function and no pertinent symptoms.

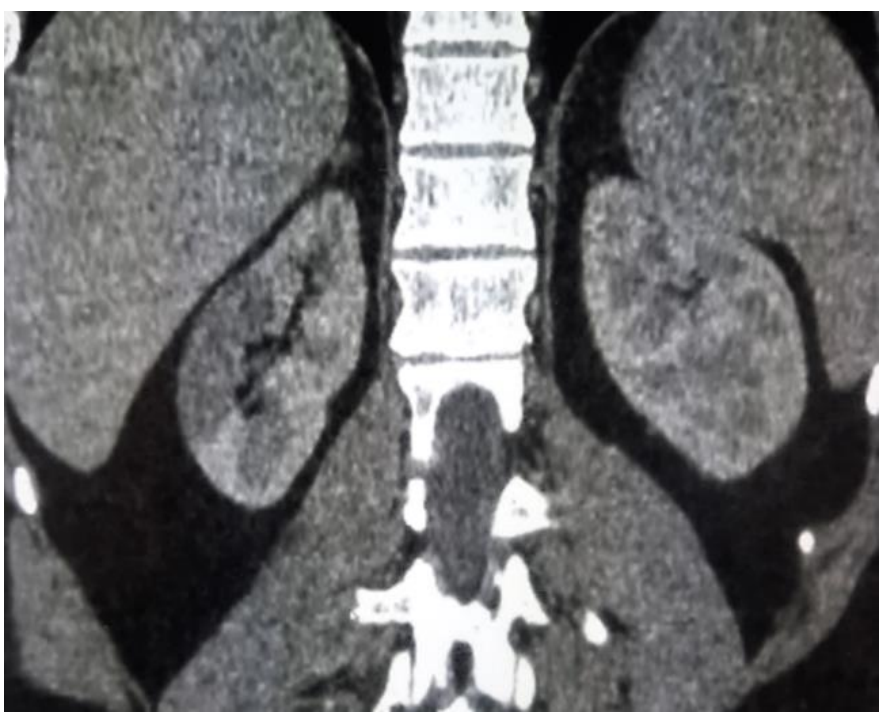

Figure 2: Contrast-enhanced coronal CT image demonstrates a wedge-shaped non-enhancing lesion in the right kidney with no perinephric inflammatory stranding

C. Case 3

A 51-year-old female underwent surgical valve repair for a severe mitral insufficiency (using cardiopulmonary bypass). There were no immediate postoperative complications. Five days following the intervention, the patient presented with acute abdominal pain and oliguria. Her vital signs were unremarkable. Physical examination was significant for tenderness in the right flank and abdominal distention.

This publication is licensed under Creative Commons Attribution CC BY http://dx.doi.org/10.29322/IJSRP.11.11.2021.p11955
There was no clinical sign of a distended bladder. Lab workup demonstrated leukocytosis at 16.200 , elevated C-reactive protein at $236 \mathrm{mg} / \mathrm{l}$, acute kidney injury (creatinine: $3.1 \mathrm{mg} / \mathrm{dl}$ from a baseline of $0.7 \mathrm{mg} / \mathrm{dl}$ ), and elevation of lactate dehydrogenase (LDH) - $827 \mathrm{U} / \mathrm{L}$ (reference value 135 - $225 \mathrm{U} / \mathrm{L}$ ). Urinalysis was positive for hematuria, whereas urine culture was negative for any infection. Echocardiography estimated left systolic ventricular ejection at $30 \%$. Acute kidney injury was initially considered as a complication of the cardiac surgery. Given the course of evolution, additional paraclinical investigations were requested by the nephrology team. Computed tomography (CT) scan of the abdomen and pelvis without contrast showed paralytic ileus but with no sign of a frank intestinal occlusion. There was no evidence of nephrolithiasis as well.

A renal doppler ultrasound was performed as an abdominal contrast CT was contraindicated. It revealed multiple triangleshaped hypoechogenic areas in both kidneys with reduced arterial flow (Fig 3). The diagnosis of bilateral renal infarction was retained, although according to the vascular surgery team's opinion, there was no indication for an interventional therapy. Kidney failure continued its aggravation with need for emergency renal replacement therapy. The patient was discharged three weeks after the intervention and continued hemodialysis in a hospital near her domicile. At 6 months follow-up, the patient had irreversible kidney damage with need for chronic hemodialysis treatment.

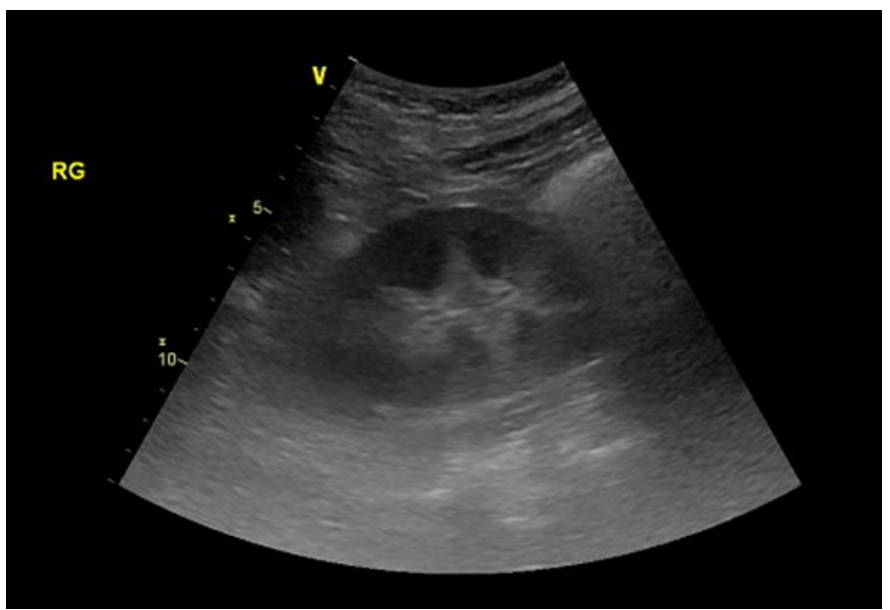

Figure 3: Doppler ultrasound with longitudinal grey-scale image showing renal ischemia as hypoechoic areas of the renal cortex

\section{DISCUSSION}

In nephrology practice, renal infarction is a very rare entity. This fact may be explained by its nonspecific clinical presentation, as it usually overlaps with more common conditions such as infectious or lithiasic kidney disease (2,3). Lab work often shows leukocytosis, elevated LDH (up to four times higher than normal) and mild elevation of transaminases and C-reactive protein $(4,5,6)$. Microscopic hematuria is frequently present, although gross hematuria is rare (7). On the other hand, acute kidney injury is a common feature at presentation or in the days following diagnosis, particularly in the case of bilateral infarction $(8,9)$. Because of all these unspecific features, it is preferable to always suspect renal infarction in the presence of acute flank pain and increased LDH 
ISSN 2250-3153

levels, especially of the patient has thromboembolic risk factors. Renal angiography is considered the gold standard exam, although the use of non-enhanced computed tomography (CT) is more common in the emergency room.

However, contrast-enhanced CT is of great importance as it contributes to the establishment of the diagnosis. The CT study may show different presentations of renal infarction: the common finding is a wedge-shaped perfusion defect, but it can also present as multifocal or global renal ischemia (10). The presence of a cortical rim sign can be noted, which is an enhanced thin layer of renal parenchyma at the periphery, due to preserved capsular circulation $(7,10)$.

Risk factors for renal infarction involve atrial fibrillation, previous, pulmonary embolism, and heart disease, whether it is ischemic or valvular (11). Therefore, etiological investigation is a vital step in the evaluation of renal infarction and must include the following exams: ECG, echocardiography, CT angiography and evaluation for prothrombotic and autoantibodies assay (12).

Because there is a lack of comparative studies, the optimal treatment for renal infarction is undetermined. Nevertheless, based on the consensus of the literature, anticoagulation with intravenous heparin followed by oral warfarin is the standard therapy protocol in this situation.

The prognosis of renal infarction usually depends more on the underlying comorbidities that on the renal infarction itself. Its most common repercussions include persistent hypertension and renal failure. Some patients will even need to be placed on a dialysis program, such as the case $3(7,13)$.

\section{CONCLUSION}

In summary, early diagnosis is of vital importance to prevent permanent loss of renal function. Clinical suspicion of renal infarction should be raised in the presence of sudden onset of abdominal pain and high lactate dehydrogenase in a patient with risk factors. Early anticoagulation is paramount to a rapid recovery.

\section{REFERENCES}

1. Tsai SH, Chu SJ, Chen SJ, Fan YM, Chang WC, Wu CP, Hsu CW. Acute renal infarction: a 10-year experience. Int J Clin Pract. 2007;61(1):62-67.

2. Javaid MM, Butt MA, Syed Y, Carr P. Acute renal infarction: an unusual cause of abdominal pain. Ren Fail. 2009;31(5):406-408.

3. Barbey F, Matthieu C, Nseir G, Burnier M, Teta D. A young man with a renal colic. J Intern Med. 2003;254(6):605-608.

4. Cabral Ribeiro J, Sousa L, Calaza C, Santos A. Acute segmental renal infarction due to factor V Leiden. Arch Esp Urol. 2009;62(6):486-488.

5. Braun DR, Sawczuk IS, Axelrod SA. Idiopathic renal infarction. Urology. 1995;45(1):142-145.

6. Yoshida T, Ikehara N, Miyabe H, Sakata S, Yajima K, Mukai S, Ohte N, et al. Two cases with renal infarction diagnosed in the early course using contrast-enhanced CT. Hypertens Res. 2004;27(7):523-526.
7. $\quad$ Korzets Z, Plotkin E, Bernheim J, Zissin R. The clinical spectrum of acute renal infarction. Isr Med Assoc J. 2002;4(10):781-784

8. $\quad$ Yang J, Lee JY, Na YJ, Lim SY, Kim MG, Jo SK, Cho W (2016) Risk factors and outcomes of acute renal infarction. Kidney Res Clin Pract: 35:90-95

9. Necmi Eren et al. Acute renal infarction in Turkey: a review of 121 cases. Int Urol Nephrol 2018 Nov;50(11):20672072

10. Wong WS, Moss AA, Federle MP, Cochran ST, London SS. Renal infarction: CT diagnosis and correla- tion between CT findings and etiologies. Radiology. 1984;150(1):201-205.

11. DomanovitisH, PaulisM, NikfardjamM ,et al. Acute renali nfarction. Clinical characteristics of 17 patients. Medicine (Baltimore). 1999; 78(6):386-394

12. Bolderman R, Oyen R, Verrijcken A, Knockaert D, Vanderschueren S. Idiopathic renal infarction. Am J Med. 2006;119(4):356 e359-312.

13. Rhee H, Song SH, Won Lee D, Lee SB, Kwak IS, Seong EY. The significance of clinical features in the prognosis of acute renal infarction: single center experience. Clin Exp Nephrol. 2012;16(4):611-616.

\section{AUTHORS}

First Author - Sabah Meryem, Department of Nephrology, International University Hospital Sheikh Khalifa, Mohammed VI University of Health Sciences (UM6SS), Casablanca, Morocco

Second Author - Khalfaoui Mohamed Amine, Department of Nephroogy, International University Hospital Sheikh Khalifa, Mohammed VI University of Health Sciences (UM6SS), Casablanca, Morocco

Third Author - El oury Hiba, Department of Nephrology, International University Hospital Sheikh Khalifa, Mohammed VI University of Health Sciences (UM6SS), Casablanca, Morocco

Fourth Author - Jabrane Fatimzahra, Department of Nephrology, International University Hospital Sheikh Khalifa, Mohammed VI University of Health Sciences (UM6SS), Casablanca, Morocco

Fifth Author - Kassimi Mariam, Department of Radiology, International University Hospital Sheikh Khalifa, Mohammed VI University of Health Sciences (UM6SS), Casablanca, Morocco

Sixth Author - Habi Jihane, Department of Radiology, International University Hospital Sheikh Khalifa, Mohammed VI University of Health Sciences (UM6SS), Casablanca, Morocco 
